\title{
Proceedings of the First Workshop on Standards for Microfluidics
}

\author{
Darwin R. Reyes ${ }^{1}$ and Henne van Heeren ${ }^{2}$ \\ ${ }^{1}$ National Institute of Standards and Technology, \\ Gaithersburg, MD 20899, USA \\ ${ }^{2}$ EnablingMNT, \\ Drakensteynlaan 34, 3319 RG, Dordrecht, The Netherlands \\ darwin.reyes@nist.gov \\ henne@enablingmnt.com
}

\begin{abstract}
In the last two decades, the microfluidics/lab-on-a-chip field has evolved from the concept of micro total analysis systems, where systems with integrated pretreatment and analysis of chemicals were envisioned, to what is known today as lab-on-a-chip, which is expected to be modular. This field has shown great potential for the development of technologies that can make, and to some extent are making, a big difference in areas such as in vitro diagnostics, point of care testing, organ on a chip, and many more. Microfluidics plays an essential role in these systems, and determining the standards needed in this area is critical for enabling new markets and products, and to advance research and development. Our goal was to bring together stakeholders from industry, academia, and government to discuss and define the needs within the field for the development of standards. This publication contains a summary of the workshop, abstracts from each presentation, and a summary of the breakout sessions from the National Institute of Standards and Technology Workshop on Standards for Microfluidics, held on June 1-2, 2017. The workshop was attended by 46 persons from 26 organizations and 11 countries. This was a unique and exciting opportunity for stakeholders from all over the world to join in the discussion of future developments towards standardization in the microfluidics arena.
\end{abstract}

Key words: cell-based assays; flow control; integration; interconnectors; in vitro diagnostics; lab-on-a-chip; microfluidics; modular; sensors; standards; testing.

Accepted: December 3, 2018

Published: January 28, 2019

https://doi.org/10.6028/jres.124.001

\section{Executive Summary}

\subsection{General Comments}

Standardization brings advantages and opportunities for research and industry, enabling interoperability of products from different suppliers. The emerging field of microfluidics, in which interconnected networks of microchannels are used to precisely control and manipulate fluids in order to carry out chemical, biological, and physical processes with applications in areas such as life science, biomedical research and biotechnology, among others, has evolved to the point where standardization will enable new markets and products and advance research and development. The attendees of the National Institute of Standards and Technology (NIST) Workshop on Standards for Microfluidics, held on June 1-2, 
2017, concurred that the agreements so far-e.g., the $1.5 \mathrm{~mm}$ grid for microfluidic ports, the $15 \mathrm{~mm}$ scheme that is the basis for the building block dimension standard, and the proposed operational classesare an excellent basis for further standardization work [1]. The guidelines that describe these standards are expected to foster fast and creative product development. Standardization would bring ample benefits, not only for developers and producers, but also for the users, by fostering competition and enabling more versatility through better mix-and-match permutations.

The lack of standardization and industry-wide-supported roadmaps make the development of products based on economic and reliable fabrication processes difficult. Standards are tools that facilitate: (1) communication for mutual understanding between customer and supplier, (2) standard protocols to normalize testing practices between companies, and (3) manufacturing practices and commerce between different customers and companies around the world. They are especially valuable for microfluidics manufacturers that develop products for low to medium volumes (up to tens of thousands per year), although testing protocols should be of interest to high-volume manufacturers as well. Attendees with a fabrication background stated that in the current situation, each customer has their own design, and reusage of existing designs by different customers is hardly possible. This situation makes production unnecessarily expensive and limits learning opportunities. This same issue also applies to test protocols. Since each product is tested with its own protocol, the results are difficult to generalize and translate back to the fabrication and design departments to improve product manufacturing and the processes associated with different stages of manufacturing, including quality control (QC).

This workshop was seen as an important step forward for the microfluidics industry and provided a venue to voice a broad view of issues regarding standardization. Ultimately, as a result of constructive and inclusive discussions, the major issues faced by the microfluidics community were identified, with the expectation that more discussions and publications will follow.

\subsection{Interconnections}

In general, there is a need for standardization of fluidic, electrical, and optical interconnections across the full range of types and sizes. This includes interfacing between micro- and macrosize scales. To be compatible with automatic dispensing units, it is necessary to adhere to the standards used by them. Therefore, existing de facto standards such as the $4.5 \mathrm{~mm}$ port pitch will continue to persist. There is the possibility that an interconnection system for the $2.25 \mathrm{~mm}$ well plate pitch will be developed as well. However, it was suggested that we should realize the limitations of well plates and that there are large numbers of applications where other microfluidic techniques are more appropriate. The $1.5 \mathrm{~mm}$ and $3 \mathrm{~mm}$ grid standards [1] appear to be acceptable for most parties, except for:

- high-volume manufacturing,

- applications focusing on high-throughput screening (HTS),

- silicon-based devices - although newly developed fan-out technologies might change that, and

- integrated products.

Combining optical and electrical connections with microfluidics is a challenge with regard to manufacturing, reliability, and cost-effectiveness. A particular problem is to separate microfluidic and electronic connections. One way of doing that is by positioning the electrical wires in plane to the microfluidic device and perpendicular to the microfluidic tubes. Another, and likely more promising approach, is to replace the tubes with a small manifold with predefined channels, reducing size and complexity. Few researchers are working with bare dies, and the small world of silicon does not fit well with the "macro-world" of microfluidics. Hence, it might be a good idea to think about components that link the silicon die to a microfluidic component in a way that (1) facilitates the microfluidic flow, (2) facilitates leak-free microfluidic connections, (3) can be handled in the average assembly line, (4) conforms to the microfluidic standards, and, if needed, (5) enables electrical interconnections. One side will be in 
contact with the silicon die, and the other will connect with the microfluidic component or system. Such a "microfluidic fan-out" needs to be affordable, reliable, and, in some cases, sterilizable.

Due to the proximity of electrical wires to microfluidic channels, leak tightness is critical, and good leakage tests are certainly needed. Microfluidic side connectors and microfluidic fan-out components may become game changers by making electrical, microfluidic, and optical interconnections to small chips and integration of silicon and microfluidics in general.

Many devices and components need microfluidic and electrical connections, so it might make sense to have standards that address a combination of electrical and microfluidic connections. Such connectors might be similar to the Peripheral Component Interconnect express card (PCI-express card) edge connectors used for electrical connections. In addition to the defined clamping exclusion area [1], a dedicated area on the microfluidic building blocks should be reserved for electrical connections, i.e., an electrical interconnection exclusion area.

Guidelines are needed to enable partners to speak one common language. They would provide details regarding design and construction requirements for electrical, fluidic, and optical connections to microfluidic devices. Standards for optical and electrical connectors are a starting point from which to establish these guidelines. Recommendations, derived in part from existing standards, were devised as follows:

\section{Recommendations List \#1}

- Formulate concepts for microfluidic fan-outs to facilitate the combination of sensors and microfluidic components.

- Identify design and fabrication needs and develop guidelines towards the standardization of connectors that combine electrical and microfluidic connections, because microfluidic and electrical connections fabricated without standardization are more likely to be physically incompatible.

- Create guidelines stipulating specific details of the design towards the inclusion of electrical connections to integrated microfluidic devices, using existing standards as the starting point.

- In addition to the already-defined clamping exclusion area, reserve a dedicated area on the microfluidic building blocks for electrical interconnections - an electrical interconnection exclusion area.

\subsection{Integration/Modular Approach}

The development phase is often where projects fail due to reliability issues or excessive development time. "Standard" components will reduce the risk of failure.

A modular approach (or modular architecture) is a proven method to address integration problems, but it needs simple, robust, and reliable connections, preferably a plug-and-play-type connection. Regarding module-to-module and module-to-world connectors, the smallest dimensions and accuracies should be based on manufacturability, reliability, and robustness, among other parameters.

The work done so far using the standard guidelines in modular systems showed that the developed standards (port pitch, building block dimensions, etc.) could be used for the design of modular microfluidic systems. A critical point in any modular approach is the actual connection of the components. This requires design guidelines and standards. Standardized dimensions (chip size and pitch) are the first (minimum) level needed to ensure interoperability and optimized development time. With these guidelines, manufacturers will be able to produce off-the-shelf components, which will lead to yield improvement and cost reductions. To reach full potential, comparable data sheets and standardized testing protocols are needed.

There is a need for miniaturized microfluidic pumps; creating them according to the proposed standard for building blocks would be an efficient approach. 


\section{Recommendations List \#2}

- Promote interoperability between suppliers so that developers and integrators can incorporate building blocks/products/connectors from different suppliers in one microfluidic assembly.

- Advocate inclusion and partnership among organizations/companies in the development of standards for interoperability; to promote the success of such partnerships, the benefits to the customers should outweigh any resistance from companies to adopt the standards developed.

- Devise strategies for comparison of products and building blocks on a generic test bench hosting generic standard test protocols; such comparison could be based on uniform material/equipment data sheets.

\subsection{Testing Protocols}

The topic of microfluidic instrument qualification was discussed, and the attendees noted that it is impossible to objectively compare products from different suppliers, because there are as yet no industrywide accepted tests, qualification protocols, or generic compliance certificates. Each company uses its own method to measure product performance. There are also no testing protocols that could help to qualify a second supplier. As most of the products and fabrication technologies are completely different, the opportunities to gain knowledge to further develop standard testing protocols are minimal to nonexistent.

Comparisons of microfluidic quality protocols used by different suppliers might be a good starting point towards the development of industry-wide accepted microfluidic test protocols. Another approach is to explore existing test protocols in use in other industry segments and assess their use or modification to comply with the microfluidic requirements. A first attempt to compile such a list was made.

However, the basis for these protocols should be the understanding of why and how microfluidic devices fail; complementary information about microfluidic device failure could be drawn from scientific publications, and so scientists should be encouraged to present this element in the dissemination of their work.

Microfluidics players should look for and work on adapted methods/protocols tuned for microfluidic testing/qualification and calibrated instrumentation.

The attendees agreed on the need for burst-test protocols and pointed to a lack of standard test protocols. These should first include accelerated lifetime testing and (nondestructive) leakage testing, including leakage test protocols for preloaded chemicals.

Several attendees mentioned the existing limitation of flow measurement techniques at the metrology institutes that measure the average flow over time. There is a great need for accurate measurement technologies for instantaneous flow measurement. The scope with regards to flow measurement should include: volumetric measurement; instantaneous measurement (including sampling frequency); Newtonian fluids; incompressible fluids; and device under test (DUT), and the results should include reference to input pressure, temperature, and output pressure.

The current work on test benches for microfluidic components is a good first step [2]; that effort should be accompanied by the following work on generic test protocols to run on such benches:

- response time, rising time, settling time;

- precision, accuracy, repeatability;

- resolution, stability;

- gas/power consumption;

- dead volumes/internal volumes/pressure drop; and

- pressure: nominal, maximal, and burst.

The next step in the standardization process could be to generate a vocabulary, i.e., definitions of properties (e.g., material properties, dimensional properties, mechanical properties, transducer properties, 
"hygiene" properties, interfacing properties, and system properties), components, and functions. As a starting point, "Micro Process Engineering - Vocabulary (ISO 10991:2009)" could be used [3].

Some new sensors have better resolution than calibrated instruments! Therefore, suitable reference sensors calibrated by external calibration laboratories or metrology institutes are also needed.

There is a need for objective, comparable methods to measure droplet sizes, especially for small volumes $(<100 \mathrm{~nL})$. The use of fluorescein could be an option.

\section{Recommendations List \#3}

- Work on extending the understanding of why and how microfluidic devices fail.

- Generate a vocabulary, i.e., a list of definitions of properties, components, functions, etc.

- List, describe, and prioritize parameters to be tested. The top three already identified are burst test, leakage test, and flow test protocols that measure instantaneous flows.

- Identify existing norms and protocols in use by the microfluidic industry.

- Ensure that suitable test benches/instruments will be developed.

- Work on reference sensors calibrated by government metrology institutes or calibration laboratories that are traceable to the national metrology institutes that correspond to and address the microfluidic state of the art.

\subsection{New Areas}

Considering the developments in organ-on-chip, and other biological applications, efforts should be made to (1) decide on the most important elements that should be standardized in these new areas and (2) hold future meetings to discuss more detailed plans for the development of such standards.

\section{Oral Presentation Abstracts ${ }^{1}$}

\subsection{Session 1: Combining Microfluidics and Electronics (Integration)}

\subsubsection{Standards for Integration of Micro-Electrical Sensors with Microfluidics: Device and Interface Design, Fabrication, and Testing}

Mark Tondra, Diagnostic Biosensors, USA

Diagnostic Biosensors (DBS) was started by the presenter (Mark Tondra) in 2005 with the purpose of integrating semiconductor sensor devices with microfluidics and biochemistry, and producing them as products. He had worked at an integrated magnetic sensor company (NVE) for about 10 years prior, where he collaborated on several federally funded research and development (R\&D) programs to develop technology that added fluidics to integrated circuit (IC) devices.

This First Workshop of Standards for Microfluidics is a great opportunity. As an example of our motivation, one of our DBS company sayings is: "We put the chip into lab-on-a-chip." This presentation is from the perspective of a small commercial technology development and production company, and its partners, which greatly benefit from standards for microfluidics and participate in standards activities.

It will start with visual examples and explanations of our still-evolving, particular DBS manufacturing and test processes to give some understanding of the practical limits of what DBS can produce. Then, designs of some IC sensor + microfluidic devices and interfaces for device-to-device connection are shown.

The final part will relate the DBS-specific designs and interfaces to published standards from SEMI (formerly known as Semiconductor Equipment and Materials International) and International Organization

${ }^{1}$ Certain commercial products or company names are identified here to describe the discussions adequately. Such identification is not intended to imply recommendation or endorsement by the National Institute of Standards and Technology, nor is it intended to imply that the products or names identified are necessarily the best available for the purpose. 
for Standardization (ISO) in the area of microfluidics integration. This part will make some estimate of the adequacy of existing standards, and of technology areas where standards will be most needed in the near future.

\subsubsection{Wafer-Level Integration of Microelectronics and Optoelectronics in Microfluidics}

Chengxun Liu, IMEC, Belgium

A large family of lab-on-a-chip devices is enabled by miniaturized active microelectronic or optoelectronic elements. For example, the well-known dielectrophoretic cell actuations or impedance-based biosensing are supported by microelectronic circuitry. On-chip diffractive optics or photonic waveguides have demonstrated the potential for high-throughput fluorescence assays, commonly used for in vitro diagnostics. The proper combination and integration of the active sensors and actuators appear to be a powerful and flexible solution towards a truly integrated lab-on-a-chip that includes all necessary bioanalytical steps, from sample preparation to reaction and detection.

However, the hybrid integration of one or more types of active components in microfluidics is rather complicated compared to passive microfluidics such as microfilters, affinity-based cell capture, or inertialflow cell isolators. Not only do the multiple types of active elements need to be compatible to each other for materials, device physics, and biochemistry, but the fabrication method should be feasible for mass manufacturing, including cleanroom fabrication and packaging.

This talk will present the efforts made by IMEC toward the selection and integration of microelectronic and optoelectronic components for lab-on-a-chip applications. One major focus is the development of wafer-level microfluidics processing, including packaging.

\subsubsection{Printed Materials and Structures for Low-Cost, On-Chip Functionalities—Prospects for Standardization}

Erik Beckert, Fraunhofer IOF, Germany

Inkjet printing of functional single- and multilayer structures provides a cost-efficient way to integrate functional structures for liquid handling and analysis on polymer or glass-based lab-on-chip systems. Printing as a versatile and digital approach allows for chip quantities from 10 to $10^{6}$, functionalities ranging from simple electrodes to heaters, sensory surfaces, and light-emitting or light-sensing semiconductor structures. An application example will be an all-printed, on-chip fluorescence detector setup that contains printed filter, organic light-emitting cells, and photodiodes. The prospects for standardization are that these structures all can be considered to be transducers, transforming electrical signals from a potentially standardized chip interface to the actual functionality on the chip.

\subsection{Session 2: Cell-Based Assays/In Vitro Diagnostics}

\subsubsection{Keynote Presentation: Microfluidics and Regulations for In Vitro Diagnostics}

\subsubsection{Standards and Food and Drug Administration Regulation of In Vitro Diagnostics}

Yun-Fu Hu, Food and Drug Administration (FDA), USA

In vitro diagnostic tests (IVDs) are medical devices subject to FDA regulation. The FDA approves a medical device based on its determination of the safety and effectiveness of the device. The device manufacturer (or sponsor of the premarket submission) is required to provide the FDA with a reasonable assurance that the probable benefits of using the device outweigh any probable risks and a reasonable assurance that the use of the device will provide clinically significant results. This evidence is determined for diagnostic tests by assessing the analytical validation (assurance of test performance) and clinical 
validation (correlation between test result and clinical condition). The FDA believes that conformance with recognized consensus standards can support a reasonable assurance of safety and/or effectiveness for many applicable aspects of medical devices. This presentation is intended to provide an overview of FDA regulation of in vitro diagnostics and discuss some regulatory challenges and opportunities for development of standards for use in regulatory decisions.

\subsubsection{Cellix's Microfluidic Solutions for Cell-Based Assays and Considerations for Standards in Microfluidics}

Vivienne Williams, Cellix Ltd., Ireland

Cellix develops microfluidics technologies for diagnostics, integrating and miniaturizing sample preparation techniques on-chip for cell-based assays that enable our customers to efficiently target point-ofcare and diagnostic solutions for multiple applications, such as: personalized healthcare, food and beverage analysis, and the agri-biotech sector. This presentation will highlight the developments of our technologies and key factors that must be considered for the development of microfluidic standards when applied to the fabrication of microfluidic chips (e.g., regulatory approved materials used in chip fabrication) and with respect to the types of samples used, the applications, and the needs of the end-user. These latter factors will explore the requirements of cell-based assays such as cell sample contact with microfluidic chip materials and currently employed ISO standards.

\subsubsection{Multi-Organ Microphysiological Platforms for Drug Testing}

Mandy Esch, Ph.D., National Institute of Standards and Technology (NIST), USA

Clinical trials for new drugs can uncover unexpected drug toxicity. Many drugs that work in animals do not produce the same results in humans. In other words, drugs that are not toxic for animals can have a very different effect in human patients. Unless we develop better mimics of the human body that can be used for drug testing, patients will continue to be exposed to drugs that will not fulfill their expectations.

Our group works on the development of multi-organ microphysiological systems that can mimic the human metabolism. Those platforms can be used to simulate drug exposure, their conversion to metabolites, and the effects metabolites have on the human body. We integrate sensors that can measure positive and adverse effects of drugs and drug metabolites continuously throughout a long-term drug exposure. We are developing a platform that contains chambers for ten tissues, mimicking ten human organs. Sensor elements are optical, electrical, and electrochemical. Together with physiologically based pharmacokinetic models (PBPK), data obtained from the platform can be extrapolated to make predictions for human patients.

The adoption of multi-organ microphysiological systems requires the systems to be inexpensive, to be easy to use, and to mimic the human metabolism as truthfully as possible. Replicating results obtained with such systems will depend on implementing standards ranging from platform materials and design to usage.

\subsection{Session 3: Flow Control and Verification Testing}

\subsubsection{Macro-to-Micro Interfaces for Microfluidic Devices}

Hugh Fan, University of Florida, USA

Since the concept of micro total analysis systems ( $\mu$ TAS) was invented, myriad microfluidic devices have been demonstrated for a variety of applications. However, an important hurdle that still needs to be cleared is the connection of a microfluidic device with the rest of the world, which is often referred to as the macro-to-micro interface, interconnect, or world-to-chip interface. In this talk, I will examine the methods used by pioneers in the field and other investigators, discuss the progresses made in past decades, 
review the approaches for electroosmotic-pumping-based microfluidic devices and pneumatic-pumpingbased devices, and present personal perspectives on the interface standardization. The advantages and disadvantages of both modular and integrated interconnects will be analyzed. In addition, past efforts in the interface standardization from the perspectives of semiconductor industries will be briefly reviewed.

\subsubsection{Standards, Guidelines, and Validation Tests for Microfluidics: Challenges from a Foundry Perspective}

Alexios Tzannis, IMT AG, Switzerland

Microfluidics, a technology characterized by the engineered manipulation of fluids at the submillimeter scale, has shown considerable promise for improving diagnostics and biology research. Currently, the state-of-the-art is characterized by the best-conduct practice of each foundry and in agreement with the immediate needs of the specific customer. On the end-user side, in order to reduce timeto-market, to stay competitive, and to keep a good yield, most of the commercialized microfluidic devices are today predominantly simple devices, using one single material with little integration. The solutions provided by the service providers are determined by the technologies available in each fabrication (polymer, glass, and silicon), leading to a lack of standardization and interoperability. Many foundries base their technology proposal on processes available from the micro-electro-mechanical systems (MEMS) industry, utilizing classical MEMS as well as non-MEMS-compatible materials, e.g., glass, gold, dielectrics. It is therefore reasonable to look at the available standardization protocols and methodologies available in the MEMS industry to address standardization and QC issues. Nonetheless, this does not address issues of deviating materials, e.g., plastics, or processes, e.g., lamination, inject printing, etc. It is the aim of this presentation to demonstrate existing and possible standardization protocols and validation tests aiming towards hybridization to use the best of each material and technology, including glass, silicon, PCB (printed circuit board), and plastics as basis materials, and appropriate processes.

\subsubsection{Microfluidics for Precision Electromagnetic Characterization of Complex Fluids at Microwave and Millimeter-Wave Frequencies}

Jim Booth, National Institute of Standards and Technology (NIST, USA)

Electromagnetic approaches provide a means to rapidly measure the physical properties of complex fluids and fluid mixtures, potentially at very high speeds. The integration of electromagnetic-based sensors with microfluidics represents an opportunity to develop novel measurement platforms to rapidly and accurately evaluate a number of different physical properties of complex fluids. The potential sensitivity of electromagnetic measurements requires, however, extremely fine control over the flow characteristics within the local environment of microfluidic structures, including flow, pressures, temperature, etc.

We present results demonstrating quantitative broadband dielectric permittivity measurements (dielectric spectroscopy) of fluids in microfluidic channels over a wide range of frequencies, from $\sim 1 \mathrm{kHz}$ up to $110 \mathrm{GHz}$. In addition to dielectric permittivity measurements, we also present recent progress in the development of microfluidic-based electron paramagnetic resonance (EPR) measurements in order to investigate the combined magnetic and dielectric properties of complex fluids at microwave and millimeter-wave frequencies.

\subsection{Session 4: Sensors, Testing, and Interconnectors}

\subsubsection{METAS - Federal Institute of Metrology to Guarantee Traceability in Liquid Flow Related TO Microfluidics}


Hugo Bissig, METAS, Switzerland

The Federal Institute of Metrology (METAS) is the national metrology institute of Switzerland. The tasks of METAS are fundamental metrology, research and development, industrial metrology, technology transfer, and legal metrology.

METAS has developed facilities for flow rates ranging from $100 \mathrm{~nL} / \mathrm{min}$ up to $400 \mathrm{~mL} / \mathrm{min}$ with uncertainties from $0.7 \%$ to $0.07 \%$ to cover the flow rates in the upcoming field of microfluidics. A more important factor for the whole flow rate range is that METAS has developed flow generators that can be operated with liquids other than water. The flow generators are homemade syringe pumps where the volume flow rates can be calibrated by means of the dynamic gravimetric method using water as liquid. These syringe pumps can then be operated with any other liquids because the volume flow rate is traceable to the International System of Units (SI). To guarantee traceable calibrations of mass flow rates, the density of the used liquid has to be determined by means of traceable density measurements in order to calculate the mass flow rate based on the measured volume flow rate.

Moreover, METAS plans to increase the range of applicable pressures up to 400 bar and temperatures between $5{ }^{\circ} \mathrm{C}$ and $50{ }^{\circ} \mathrm{C}$. In a further step, METAS intends to reorient the activities of the flow laboratory towards the application and characterization of complex fluids.

In another area, METAS has experience in the conformity assessment of various instruments that are under legal control. The assessment requires tests to be performed according the corresponding normative document or standards. METAS is working within different committees that set up or review these standards.

\subsubsection{Microfluidic Flow Control and Monitoring for Characterization and Validation}

Anne Le Nel, William Cesar, Fluigent, France

Currently, there is a need to define standards in microfluidics, with the aim of bringing benefits to the industry and achieving plug-and-play microfluidics. For its own production line, Fluigent had to adapt the standard validation protocols and materials to characterize its microfluidic instrument range. In a recent effort towards this standardization, Fluigent contributed its 11 years of expertise in microfluidic instrumentation by joining other major actors and academic institutes in the MFManufacturing Consortium. In this project, standards addressing component geometries, clamping, and interconnections were defined. Modular microfluidic systems were built on this basis using microfluidic building blocks (MFBB) such as valves, reactors, mixers, reservoirs, etc. This raised questions: How should the fluidic characteristics of these elementary building blocks be measured? How can their correct behavior be assessed? How can it be demonstrated that two given types of MFBB have comparable functions and are interchangeable? Those questions are essential both to the manufacturer for quality check and to the end-user for design purposes.

Fluigent combined its know-how in microfluidic instrumentation, integration, and software design by engineering integrated generic characterization benches to test fluidic properties of any MFBB using performant flow control instruments (pressure pump, flow sensors, valves). Specific care was given to ergonomics, robustness, metrology, and automation. Dedicated software was developed for flow automation and computation of fluidic characteristics such as hydrodynamic resistance or closing behavior of pneumatic valves and many more.

\subsubsection{Standards and Testing-Addressing the Accuracy and Precision of "Small"-Volume Transfers in Droplet-Based Microfluidics}

Sammy S. Datwani, Labcyte Inc., USA

Emerging assays (biochemical, enzymatic, cell-based, polymerase chain reaction [PCR], qPCR (quantitative PCR), dPCR (digital PCR), ddPCR (droplet digital PCR), etc.) in droplet microfluidics require the measurement of a variety of parameters such as droplet size, droplet volume, droplet concentration, 
droplet velocity, droplet trajectory, droplet shape, and others. The characterization of droplets is critical to the development of accepted standards for droplet-based microfluidics, and even though many techniques exist, such as high-speed imaging, photometry, pressure sensing, calorimetry, radiolabeling, gravimetric methods, amplification methods, fluorescence, ratiometric methods, hybrid methods, and others, there is no one accepted standard for addressing "small" (nanoliter or picoliter) volumes.

This study aimed to assess the suitability of utilizing sodium fluorescein to measure the precision and accuracy of fluid transfers of $2.5 \mathrm{~nL}$ droplets of aqueous (water, glycerol, Triton-X 100) or dimethyl sulfoxide (DMSO)/water solutions (70-100\%) to quantify the accuracy and the precision and present a robust process that could be deployed for measuring droplet volume accuracy and droplet volume precision. The short-term and long-term stabilities of sodium fluorescein were qualified with a reference standard. Next, the noise contribution from the bulk-filler and the fluorescence reader were determined. Finally, results were presented for the accuracy and precision of 5 and $50 \mathrm{~nL}$ fluid transfers using sodium fluorescein as the measurement process.

\subsection{Session 5: Modular Systems}

\subsubsection{Reconfigurable Modular Microfluidic Systems}

Po Ki Yuen, Corning, USA

Reconfigurable modular microfluidic systems that can be assembled, disassembled, reconfigured, and assembled again to build different integrated microfluidic systems were presented. Each microfluidic module can be designed and optimized separately before connecting them together to form a larger system. The modular design approach or modular architecture is one of the proven approaches to address the integration problem when developing multifunction microfluidic systems. In addition to real-world applications, the reconfigurable modular microfluidic systems with basic microfluidic technologies could be a very useful tool in teaching laboratories with limited resources for expensive and high-technology equipment and will lower the barriers for new entrants to the field of microscale devices and systems.

\subsubsection{Complex Microfluidic Devices: Modular Approach and Hybrid Solution}

\section{Nicolas Verplanck, CEA-LETI, France}

In the microfluidics market, the point-of-care diagnostics application remains the main driver in volume, representing $60 \%$ of the market today in units and only $10 \%$ in value. This application is driven by costs and mass production. Other applications, such as pharmaceutical and life science research or analytical devices, constitute the main driver for highly complex and functional microfluidic devices, with high added value and low volumes.

However, a complex microfluidic device is rarely possible in a single injection molding step and requires the integration of specific functions such as sensors, e.g., MEMS, and actuators, e.g., active valves, fabricated in different materials (glass, silicon, polymers). Moreover, microfluidics manufacturers are mostly specialized in one of the predominant types of basic materials (glass, silicon, and polymer). The most reliable and cost-efficient solution is to adopt a modular approach by using heterogeneous components from different manufacturers.

This presentation focused on top connections and presented some examples of complex microfluidic devices developed at CEA-LETI and in the European MFManufacturing project.

\subsubsection{Standardized Modular Microfluidic Edge Connections}


Wilfred Buesink, Micronit, The Netherlands

As the maturity of microfluidic applications grows, the demands and restrictions on interfacing methods also grow. The most common fluidic interfacing connections are based on a top-down clamping approach. In many cases, aspects like dead volume, shear rate, reduced device footprint, or maximum optical access are critical, and a planar connection approach can be beneficial. The latest advances in microtechnology and related manufacturing technologies are enabling good-quality modular edge connections for a wide range of microfluidic chips.

Today's market is no longer satisfied with a standard or a specific custom solution; the market requires both. The standardization created within the MFManufacturing project provides the interfacing boundaries and interoperability between companies to the user while leaving the specific solution and requirements open for the design house. This has also been the vision for interfacing solutions offered by Micronit, making the currently available solutions easily compatible with the created standard for both top-down and edge connections.

The presentation provided insight into the ways in which modular edge connect standards enable improved application quality and ease-of-use, as well as giving a roadmap towards the next-generation solutions.

\section{Remarks from the Individual Presentations}

\subsection{Opening Session}

Welcome and opening remarks were made by Dr. Laurie Locascio, director of the Material Measurement Laboratory and acting Associate Director for Laboratory Programs, NIST. Dr. Locascio welcomed the attendees and stressed the importance of standards in general. One key remark she made was that standards don't hold back innovation; they promote it.

Dr. James Olthoff, director of the NIST Physical Measurement Laboratory, gave opening remarks on the second day.

\subsection{Overview of the International Workshop Agreement on Interoperability of Microfluidic Devices-Guidelines for Pitch Spacing Dimensions and Initial Device Classification Henne van Heeren, enablingMNT, The Netherlands}

\subsubsection{Introduction}

Integration of microfluidic components from different suppliers into one system often requires ad hoc solutions, leading to reliability problems. Industry agreements about interconnections and component formats would simplify matters for designers and producers of microfluidic devices, especially if industrywide-supported quality standards are produced. Such agreements will facilitate an easier and more reliable way to work with microfluidic devices (e.g., will lead to plug-and-play microfluidics). A particular problem in the microfluidic industry is the vast variation in technologies and materials used. Therefore, to make the standards widely applicable, they should be formulated in such a way that they are independent of technology and application. A multinational group of microfluidic companies and institutes has agreed on such specifications [1].

\subsubsection{Microfluidic Interconnections}

The first problem discussed by the multinational group was the issues of microfluidic interconnections. These are often made manually, and when many connections are needed, this could become a costly activity with an increasing number of reliability issues. To promote the introduction of easy-to-use multiport connectors, the positions, sizes, and nomenclature of microfluidic ports were defined (see Fig. 1 and 2). 


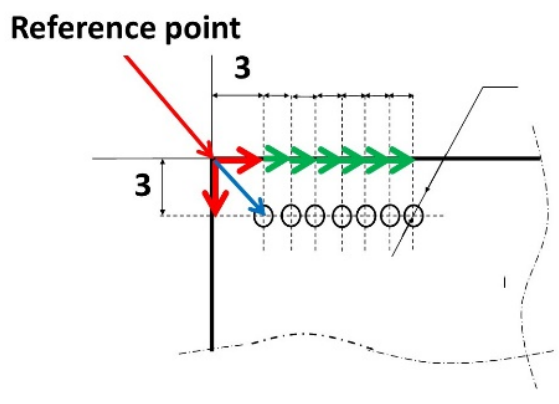

Fig. 1. Microfluidic port positions and port nomenclature, where the center of the first port is at $(3 \mathrm{~mm}, 3 \mathrm{~mm})$ from the reference point, and the pitch between port centers is $1.5 \mathrm{~mm}$.

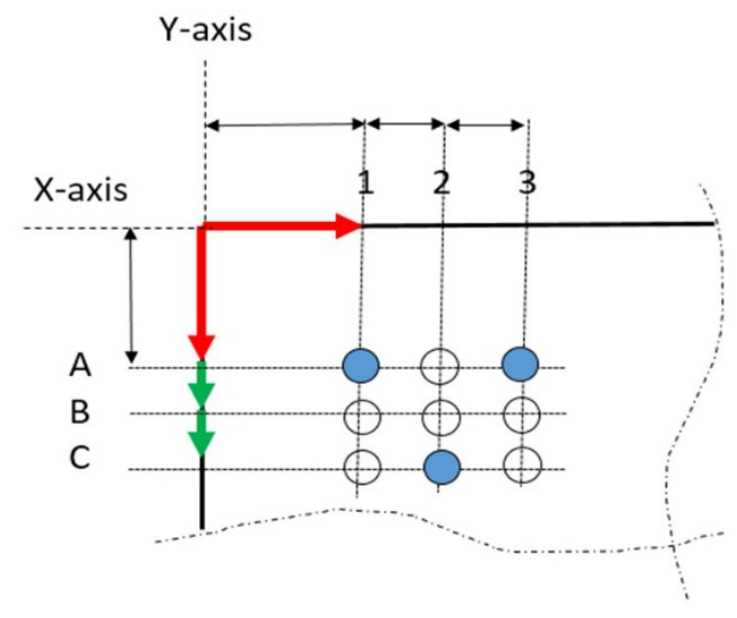

Fig. 2. Microfluidic port nomenclature, with ports $\mathrm{A} 1, \mathrm{~A} 3$, and $\mathrm{C} 2$ shown in blue on a $2.5 \mathrm{~mm}$ port grid.

The second important step the group took was defining dimensions of standard microfluidic building blocks to enable easy assembly of the components. This could also enable interchanging parts, for instance, to use components from different suppliers or to create a reconfigurable system. In discussion are guidelines about side connectors and microfluidic circuit boards. It is good to mention that all the guidelines were only approved after thorough discussions with dozens of companies outside the core group.

\subsubsection{Towards Generic Validation Testing for Microfluidics}

The third step taken was defining a classification of microfluidic devices; this is seen as an essential step towards industry-supported quality specifications. Although at first this classification only addresses pressure and temperature operation ranges (see Table 1), they are to be complemented by flow ranges. 
Table 1. Operational classes for microfluidic devices.

\begin{tabular}{|c|c|c|c|}
\hline Class Type & $\begin{array}{c}\text { Maximum Pressure } \\
(\text { bar })\end{array}$ & $\begin{array}{c}\text { Maximum } \\
\text { Temperature }\left({ }^{\circ} \mathrm{C}\right)\end{array}$ & $\begin{array}{c}\text { Minimum } \\
\text { Temperature }\left({ }^{\circ} \mathrm{C}\right)\end{array}$ \\
\hline PT $2 / 50$ & 2 & 50 & 4 \\
\hline PT $2 / 75$ & 2 & 75 & 4 \\
\hline PT $2 / 100$ & 2 & 100 & 4 \\
\hline PT $7 / 50$ & 7 & 50 & 4 \\
\hline PT 7/100 & 7 & 100 & 4 \\
\hline PT 30/50 & 30 & 50 & 4 \\
\hline
\end{tabular}

The rationales for this part of the discussion are the following: (1) There are no published generic test protocols based on proven fault modes to assist the development of more reliable microfluidic products. (2) Most of the tools and techniques currently used for failure analysis are leveraged from the IC industry, and they are not designed to be used with fluids, especially not with liquids. Therefore, the microfluidic industry faces the challenge that it needs to define its own testing strategies, methods, and reliability models. These testing strategies should be based on the most challenging microfluidic fault modes. Hence, identifying and understanding these fault modes are factors of major importance for the microfluidics community.

\subsubsection{White Papers and Guidelines}

The proposed standards and guidelines will be published as white papers to ensure wide dissemination. Several of the standards developed so far are currently being transferred to the International Standard Organization (ISO) to become official standards. The guidelines proved to be very easily applicable for designers, and within 2 years after publication of the first guideline, about 15 new products based on these standards are in development. As one of these products already needed further miniaturization, we discussed future (smaller) formats too.

\subsection{Session 1: Combining Microfluidics and Electronics (Integration)}

Mark Tondra, Diagnostic Biosensors, USA

Mark presented the SEMI activities on standardization of microfluidics. This work was driven by the wish to introduce microfluidic capabilities to the traditional electronic industry. A particular problem here is the separation of microfluidic and electronic connections. One way of doing that is to position the electrical wires perpendicular to the microfluidic tubes. Another and likely more promising approach is to replace the tubes with a small manifold with predefined channels, reducing size and complexity. One cannot use a microfluidic chip if it does not fit onto something, so the microfluidic manifold might be used for that. Due to the proximity of electrical wires to microfluidic channels, leak tightness is critical, and sound leakage tests are certainly needed. Sealing technologies that can be used include reusable ones, such as compression gaskets and O-rings, or permanent ones based on glue, adhesive, or thermal bonding, welding, etc.

Other problems that are slowing down microfluidic developments are (1) the lack of maturity in technologies used to stick several layers together; and (2) stress on the fragile electronic structures, but this likely can be solved by using flexible circuit boards.

Edge connectors used in the electronics industry might provide ideas on how to combine electronic and microfluidic interconnections in one connector. 
Chengxun Liu, IMEC, Belgium

Chengxun addressed the issue of combining optical, microfluidic, and electrical connections. It turned out that it was challenging to translate the successful semiconductor approach to microfluidics. The lack of standardization and industry roadmaps makes developing products based on economic and reliable fabrication processes difficult. The diversity in applications and technologies makes it hard to find the right topics to standardize. One route forward might be wafer-scale processing; however, microfluidic channels and optical waveguides need large "real estate." In semiconductors, silicon "real estate" is expensive, and one would want to make the chips as small as possible. This small size makes them difficult to combine with the much larger microfluidic components.

Another approach is to combine PCB with microfluidics. This combination partially overcomes the constraints related to space when combining electrical, microfluidic, and optical interconnections. One solution is to divide them over the three axes: reserve left and right for electrical connections; assign front and back to optical connections; and keep the bottom for microfluidic connections.

Some other challenges are the influence of packaging technology on biosensor performance, alignment, auto-fluorescence, and control of surface properties. Some of the requirements for fabrication and packaging are:

- no damage to immobilized antibodies during packaging (e.g., room-temperature bonding);

- good alignment to the rest of the microsystem;

- little autofluorescence from the packaging material; and

- controllable physical (hydrophilicity) and chemical characteristics (modest molecule absorption).

Lessons that can be learned from the semiconductor industry include roadmap creation and consideration of ecosystems.

Determined by applications, the choice of technology and the sensitivity to cost differ substantially. It is challenging to directly unify the physical layout of a silicon-cartridge interface. However, it may be possible to find a few generic solutions for the interface.

Packaging/integration methods should preferably support:

- wafer processing,

- low-temperature bonding, and

- one side $v s$. two-side packaging.

During the discussion, it was mentioned that microfluidic side connectors might become a game changer for combining electrical, microfluidic, and optical interconnections.

Erik Beckert, Fraunhofer IOF, Germany

According to Erik, three-dimensional (3D) printing could change the microfluidic industry by offering more flexibility and design freedom, but also by providing a good solution for small volumes. This speaker stressed the need to think about the best options for electrical connections to microfluidic devices: i.e., geometry and defining electric parameters.

\subsection{Session 2: Cell-Based Assays/In Vitro Diagnostics}

\subsubsection{Keynote Presentation: Microfluidics and Regulations for In Vitro Diagnostics}

Yun-Fu Hu, Food and Drug Administration (FDA), USA

Yun-Fu gave a valuable overview of the classification of medical devices and the process to achieve FDA approval. The FDA regulations are mainly related to application issues. Whenever possible, the FDA will refer to existing standards, including from ISO.

Takeaway messages:

- FDA regulates medical devices/in vitro diagnostics based on risks to patients and also:

o intended use as a determinant of device risk classifications, 
o analytical performance (e.g., limit of detection, accuracy, precision), and

o clinical performance (e.g., sensitivity, specificity).

- FDA recognizes and uses existing standards where available for regulatory decisions.

- There are many areas in need of standards (e.g., next-generation sequencing, NGS).

- FDA encourages and participates in standards development.

Vivienne Williams, Cellix Limited, Ireland

A challenge is the translation of application requirements (fitness for use) into manufacturing processes that enable quality control. Vivienne stated that there is a relation between the (chip) material/fabrication process and the choice of microfluidic connector. Cellix' connector is based on $4.5 \mathrm{~mm}$ pitch; the utilized technology (O-rings and needles) ensures a very reliable connector that can withstand high pressures. The fact that injection molding can create accurate holes makes this possible. The connectors can be cleaned and reused. Vivien presented a list of quality checks that are used in Cellix' production.

Luis Fernández Ledesma, microLIQUID S.L., Spain

Luis stated that in the current situation, each customer seems to have its own design, and the reuse of existing designs is hardly possible, making production unnecessarily expensive and limiting learning opportunities. Chip-to-system connections are particular challenges. Another challenge is the control of a microfluidic system. The questions Luis asked the community were: (1) Should small production volume be left aside? (2) Should the control system be considered in the standardization of microfluidic cartridges? (3) Standardization would bring a lot of benefits, but mostly for developers; are we ready to provide benefits to clients?

\section{Mandy Esch, NIST, USA}

Mandy addressed issues for standardizing multi-organ platforms, where the relative sizes of organ compartments, the distribution of blood surrogate, and fluid residence times influence the data obtained for a drug exposure. She presented a list of items that should be considered for standardization of multi-organ microphysiological devices (Table 2).

Table 2. Elements to consider in body-on-a-chip standardization.

\begin{tabular}{|c|c|c|}
\hline Microfluidic Platforms & Authentic Tissues & Drug Efficacy + Side Effects \\
\hline Organ chamber size ratios & Cells & Experiment design \\
\hline Flow rates & Microenvironment & Pipetting \\
\hline Liquid-to-cell ratio & Serum & \\
\hline Nonmetabolizing tissues & Common medium & \\
\hline
\end{tabular}

\subsection{Session 3: Flow Control and Verification Testing}

Hugh Fan, University of Florida, USA

Hugh presented an overview of the history of microfluidic interconnections, leading to the easier and more reliable connection technologies that are currently used. In general, there is a need for micro-to-macro interfaces. He stressed the fact that if an application needs to be compatible with automatic dispensing units, then they need to adhere to the standards that are used in that industry. That means that, for example, the $4.5 \mathrm{~mm}$ port pitch, which is a recognized standard element there. It is possible that someone will develop an interconnection system for the $2.25 \mathrm{~mm}$ well plate pitch. One attendee noted the limitations of 
well plates and added that there are many applications were other microfluidic techniques are more appropriate.

During the discussions, the mechanisms of microfluidic device failure were considered to be important factors, and scientist should publish more about their failures.

The $1.5 \mathrm{~mm}$ scheme fits well with a well plate spacing up to 384 well plates but not for plates with higher numbers of wells.

To go forward, meetings are beneficial, and reports must be inclusive and scientifically sound.

Alexios Tzannis, IMT, Switzerland

There are currently no testing protocols that could help to qualify a second supplier. Vocabulary definitions are needed. If all the products and fabrication technologies are completely different, there is no room for a learning process that will allow this community to avoid repeating mistakes and become more efficient. Alexios pointed towards existing standards in use in other industry segments that could be used or translated into the microfluidic industry.

Next steps in the standardization process could be to generate a vocabulary, i.e., definitions of properties, components, and functions. As a starting point, "Micro Process Engineering-Vocabulary (ISO 10991:2009)" could be used [3]. Furthermore, some of these definitions should be, for example, for material properties, dimensional properties, mechanical properties, transducer properties, "hygiene" properties, interfacing properties, and system properties. A list and description of parameters to be tested and existing norms should be made that can be referenced to reduce work load; there is much overlap with other market segments. A first attempt to make such a list was presented.

With regard to testing protocols, primary goals included accelerated life-time testing and (nondestructive) leakage testing.

\section{Second Day of the Workshop, Friday, June 2, 2017}

\subsection{Session 4: Sensors, Testing, and Interconnectors}

Hugo Bissig, METAS, Switzerland

METAS and others have defined methodologies for accurate measurement of microflows $(100 \mathrm{~nL} / \mathrm{min}$ to $1 \mathrm{~mL} / \mathrm{min}$, at room temperature and pressures between 0 and 8 bars) in the operating regime where most microfluidics function.

Measurement protocols that, according to METAS, could be of interest are: (1) estimation of channel cross section using accurate flow measurement; (2) burst tests; (3) changing flow rates; (4) ability to test in the temperature range 5 to $50^{\circ} \mathrm{C}$; and (5) complex flows.

The audience agreed on the need for burst test protocols, but they also pointed out the need for leakage test protocols. Several attendees mentioned the limitation of instantaneous flow measurement techniques at the metrology institutes that measure the cumulative flow over time. There is a need for accurate measurement technologies for instantaneous flow measurement.

\section{Anne Le Nel, Fluigent, France}

Anne addressed the issue of microfluidic instrument qualification, including characterization during development, qualification of the product, and quality control in production. These activities should lead to compliance certificates, product certification, application/technical notes, and technical data sheets. Fluigent is interested in definitions/vocabulary, methods/protocols, calibrated instrumentation, and other standards. Items that should be addressed are:

- response time, rising time, settling time;

- precision, accuracy, repeatability;

- resolution, stability; 
- gas/power consumption;

- dead volumes/internal volumes/pressure drop; and

- pressure: nominal, maximal, and burst.

Adapted methods, protocols dedicated to microfluidic testing, and qualification and calibrated (metrology) instrumentation are needed. Anne pointed out that some new sensors have better resolution than calibrated instruments! It is difficult to compare products because all manufacturers seem to use their own methods to measure their product's performance.

A particular problem is that the microfluidic community is working in a wide area of parameter ranges, for example:

- pressure: from $8 \mu$ bars to 10 bars;

- flow rate: from a few nanoliters per minute to several milliliters per minute; and

- volume: from a few picoliters to several milliliters.

Fluigent is working on several issues relevant to the microfluidic community, such as reference sensors calibrated with external governmental standards institutes, internal procedures and protocols (some of them adapted from macro- to microscale), and an integrated platform (instrument) for microfluidic device testing/validation. On such an instrument, the user can run the standardized test protocols on components. Component placement in the instrument should be simple to save time and to ensure proper testing. Multiphysics characterization platforms for microfluidic systems like these will provide functional information about fluidic, electrical, and thermal behavior, among other parameters. This platform can be used for calibration, comparison between suppliers, reliability testing, etc. Now standards must be defined for characterization in terms of hardware, software, and methods.

Comments from the audience included the need for (1) miniaturizing microfluidic pumps and (2) leakage test protocols for preloaded chemicals.

Sammy Datwani, Labcyte, USA

High pressures are another driver for side connectors. There is no need to reinvent the wheel; other standards can be adapted and used. There is a need for objective, comparable methods to measure droplet sizes, especially for low volumes $(<100 \mathrm{~nL})$. The use of fluorescein might be an option.

\subsection{Session 5: Modular Systems}

Po Ki Yuen, Corning, USA

A modular approach or modular architecture is a proven strategy to address integration problems, but it needs simple, robust, reliable, and preferably plug-and-play connections. Po Ki demonstrated two such interconnection systems. These connections were tested by him using burst tests and pull force testing.

The developed interconnects can be used in microfluidic circuit boards, where building blocks having different functionality can be combined in reconfigurable systems.

The speaker listed some considerations for standardization. For module-to-module and module-toworld connectors, the dimensions should be based on manufacturability, reliability, and robustness. Locations and numbers of inlet and outlet openings should be described. Functional tests needed are: (1) leakage test at the specified pressure ranges, and (2) reliability and robustness test for repeatedly connecting and disconnecting components. For some connections, existing standards can be used (for instance, the ones used in optical connections).

\section{Nicolas Verplanck, CEA-LETI, France}

CEA-Leti and others have developed five different microfluidic circuit boards and an even larger number of components that conform to the established standards, but the need for standards to ensure compatibility and interoperability was highlighted. For instance, in the modular approach to microfluidics, a critical point is the connection between the parts. This critical issue requires design guidelines and 
standards. Standardized dimensions (chip size and pitch) are the first (minimum) level to ensure interoperability and optimize development time. The guidelines outlined herein will help manufacturers to produce off-the-shelf components, which will lead to cost reduction and yield improvement. This is only possible if comparable data sheets and standardized test protocols are available.

The new guidelines depicted here describe chip sizes, the position of microfluidic ports, and more. There are also guidelines for building blocks. Some building blocks have been made and are in the process of being designed. To test and qualify these building blocks, a test-bench has been developed (see Anne LeNel's Abstract and Notes).

Wilfred Buesink, Micronit, The Netherlands

Standardization brings advantages for research and industry, enabling interoperability of products from different suppliers. The guidelines that describe these standards are a stimulation for fast and creative product development. The community is now working to overcome challenges in setting the standards and proving the reliability of products based on these standards.

While Micronit was involved in developing the new standards (e.g., interconnectors), they were able to develop one of the first new products based on these standards (see Fig. 3).

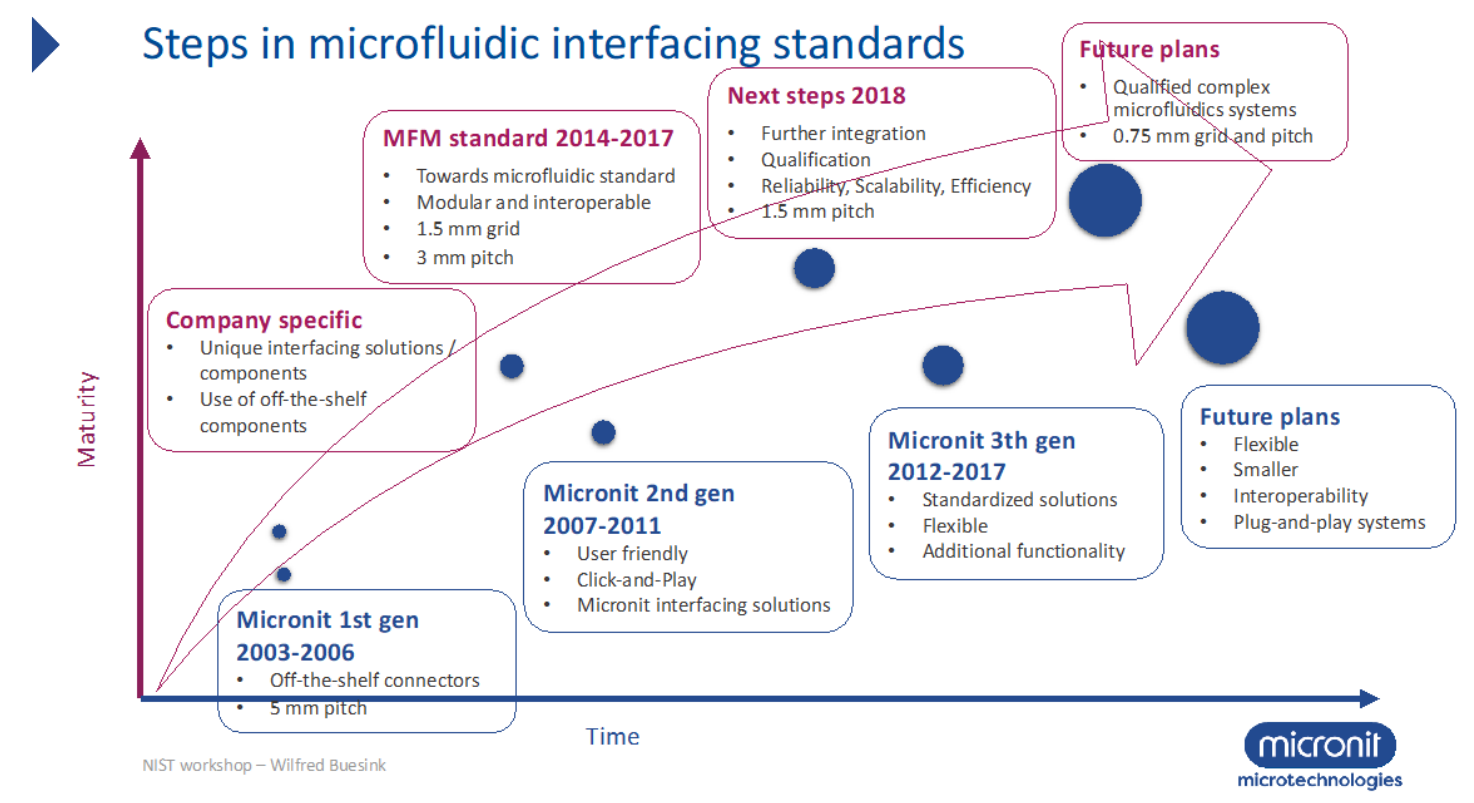

Fig. 3. Example of how standard development and new product creation go together. MFM indicates MFManufacturing project. 


\section{Journal of Research of the National Institute of Standards and Technology}

One of these products is the side connector. [During the discussion, it turned out that the terms "side connector" and "edge connector" are used for the same type of component, although they do not mean the same thing.] These side connectors have several advantages, although top connectors might be a better choice for a few cases (Table 3).

Table 3. Pros and cons of side connectors.

\begin{tabular}{|l|l|}
\hline Pro & Con \\
\hline Smooth access to microfluidic channels & Good practices in top-down assembly \\
\hline Uninterrupted flow path with minimal shear & Top-down stacking that works in the $z$-direction (large surface) \\
\hline $\begin{array}{l}\text { Low dead volume at inlets and outlets, resulting in reduced } \\
\text { sample and reagent use }\end{array}$ & $\begin{array}{l}\text { Tolerances more critical and demand on (local) cartridge } \\
\text { interface design increased }\end{array}$ \\
\hline Smaller footprint and simple fabrication & Increased demands on fabrication and/or separation methods \\
\hline $\begin{array}{l}\text { Minimized interface, maximized optical access; compatible with } \\
\text { upright and inverted microscopes }\end{array}$ & Currently not that many companies offering planar solutions \\
\hline Autoclavable and easy tube mounting & \\
\hline
\end{tabular}

Overall, side connectors have so many advantages that they will be increasingly used in the industry. Wilfred showed that several companies already offer side connectors. They differ in design, are not always easy to use, and are not affordable. There is now a guideline being written that standardizes the positions of the microfluidic ports in a similar way as the top connectors (Fig. 4). This guideline, proposed by the MFManufacturing consortium, will be available shortly.

\section{References and sizes}

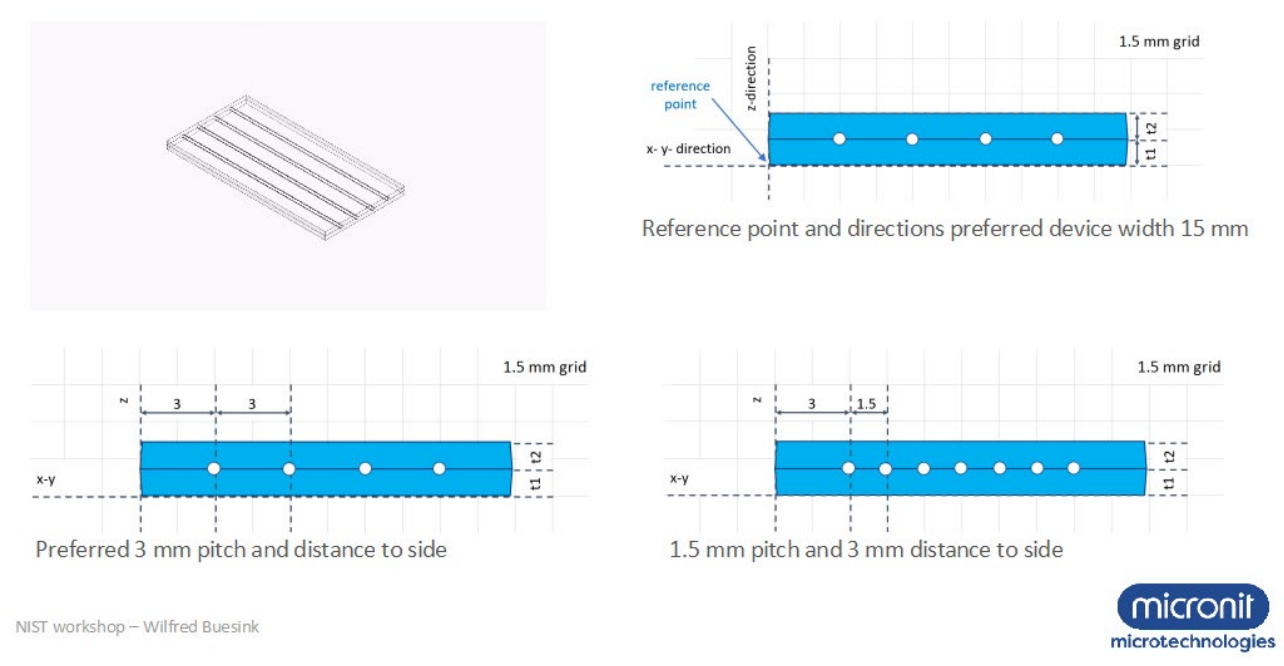

Fig. 4. Proposed standard for side connector.

Standards are particularly valuable for those that develop products in the range of low to medium volumes (up to 10000 per year). 


\section{Conclusions and Outlook}

\subsection{Integration of Microfluidics and Electronics}

Besides the already defined clamping exclusion area, a dedicated area on the microfluidic building blocks should be reserved for electrical interconnection: an electrical interconnection exclusion area.

Many devices and components need both microfluidic and electrical connections, so it might make sense to think about a standard for a connector that combines them both. Such a connector might be similar to the PCI-express card edge connectors in use for electrical connections in microelectronic systems (see Fig. 5).

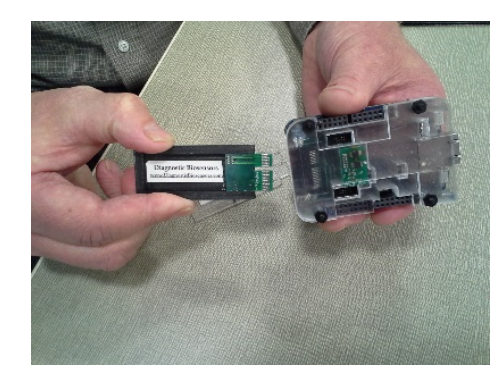

Fig. 5. Inspiration for connectors that combine microfluidic and electrical connections.

A good practice for a design engineer would be to have a list of materials that can be used in the wetted path of the device/component. There are limitations to the kind of materials that are acceptable for microfluidic designers for the wetted path. Glass, silicon, cyclic olefin copolymer/cyclic olefin polymer (COC/COP), polymethyl methacrylate (PMMA), polycarbonate (PC), and polystyrene (PS) are the most often used materials. Materials that are commonly used in the electronic industry (e.g., potting material) might leach out unwanted chemicals or carry a risk of unwanted absorption, and therefore they should not be allowed in the wetted path.

Bonding glass to silicon at low temperatures is possible, and the expansion coefficients of the two materials are such that a reliable bond is achievable. This is especially important for biosensors and other elements that contain biomaterials. In these cases, room-temperature bonding is an essential requirement during the production processes.

Few manufacturers work with bare dies, and the small world of silicon does not completely fit in with the more macroworld of microfluidics. Therefore, components are needed that "transform" the silicon die into an element that: (1) adheres to the microfluidic standards; (2) facilitates leak-free microfluidic connections; (3) can be handled in the average assembly line; (4) facilitates the microfluidic flow; and (5) enables electrical interconnections. One side will be in contact with the silicon, and the other will be in contact with the microfluidic system. Such a "microfluidic fan-out" should be affordable and reliable, and, in some cases, sterilizable.

\subsection{Flow Control, Sensors, and Testing}

Flow measurement is seen as an important aspect in standardization by companies and other stakeholders of metrology laboratories. The scope of the development of standards in flow measurement should include: volumetric measurement; Newtonian fluids; instantaneous measurement (sampling frequency or delta time); incompressible fluids; and device under test (DUT; not internally); and reference should be made to pressure and fluid temperature, and output pressure. On the other hand, the scope of the work on standards testing should focus on: standard protocols; test benches/instruments; environment 
conditions (e.g., pressure, temperature, humidity, etc.); validation (number of measurements or cycles; errors: systematic, random, etc.); and traceability (transfer standards). Finally, the scope of the work on sensors should first look at integrated sensors (e.g., optical, electrical, temperature gradient, surface acoustic wave, time of flight) or external sensors; traceability; repeatability and reproducibility; hysteresis; sensitivity to other parameters (e.g., temperature, angle, electromagnetic interference); and compatibility with fluids.

\subsection{Interconnectors and Modular Systems}

The consensus obtained in the International Workshop Agreement (IWA 23) [1] demonstrates that the $1.5 \mathrm{~mm}$ grid is acceptable to most parties. However, there are industry sectors where these interconnects will have difficulty being accepted. Some of these are: high-volume manufacturing, applications focusing on HTS, silicon-based devices (although fan-out approaches might change that), and integrated products. In these areas, silicon chips are often used in combination with microfluidics. Silicon chips tend to be rather small, and smaller port pitches might be needed. Several new activities will need to be taken up to get closer to standards in this important area. Some of these activities are:

- comparison of products and building blocks on a generic test bench, so that they will have comparable data sheets, including reliability testing and standard testing protocols;

- achieving interoperability between suppliers, so developers and integrators can use building blocks/products/connectors from different suppliers;

- development of standard components to reduce the likelihood of failure, which often occurs in the development phase due to unreliable products or lengthy development time; and

- standardization of the nomenclature related to interconnects.

\subsection{Outlook}

A wave of standardization efforts is under way by companies, academics, and metrology laboratories interested in bridging the gap between academic and industry research and the development of commercial products. Academic ideas that show promise as commercial products could be transferred faster to manufacturing if research development is done using existing and proven systems in the industrial realm, or if these products are further developed using mass manufacturing concepts. The development of standards is expected, in time, to improve the quality and reduce the cost of microfluidic components by making available standard parts and processes. The areas covered during the First Workshop on Standards for Microfluidics are just the beginning regarding the future of standardization for microfluidic systems. More and more stakeholders are getting involved in these efforts. The European consortium MFManufacturing made major progress in the last few years towards understanding the interests of industry and academia with regard to the most used components and conditions for common microfluidic applications. MFManufacturing also pressed for the development of ISO standards in microfluidics (e.g., ISO IWA 23), and more ISO standards are under development. Ongoing efforts are also growing within the Microfluidics Association (MFA), a new international organization that promotes, among other things, a common language, definitions, and standards in microfluidics.

MFA is working towards the creation of working groups on the four areas presented in this article. The first standards under development are focused on physical measurements, such as flow, dimensions (spacing between interconnects), and testing protocols. However, with the continuous expansion of biomedical applications/devices, future standards will require defining parameters of a biological nature within these systems. Generic media, standard cell microenvironments, and standard tissue models are just some of the many parameters that will likely need to be standardized in the future, when devices that include cells and other cellular-related components make it to the FDA for approval. If the efforts reported in this article persist, there is no doubt that the microfluidics field will soon have readily accessible and 
practical standards for these applications. The purpose and expectation of the companies, academics, and metrology laboratories involved in this effort are to provide the microfluidics field with standards that will ultimately benefit everyone in the field by: making results from research and development more accurate (e.g., standardized flow measurements, and commercial products with data sheets that are SI traceable); reducing the time between academic and industry research and viable commercial products (Fig. 6); achieving harmonization of terms; and defining the minimum parameters needed to qualify instruments and components as suitable for a specific application.

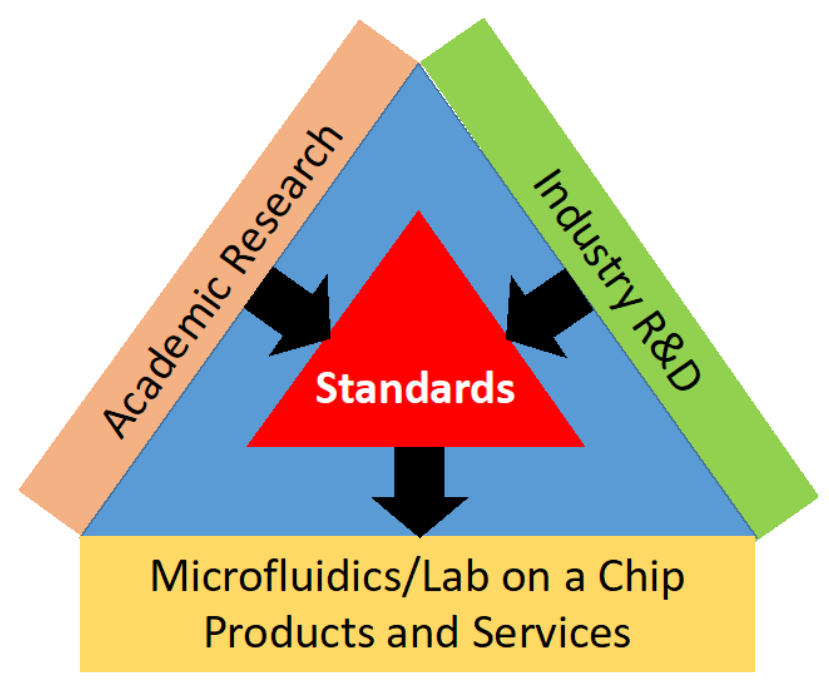

Fig. 6. Diagram of the building blocks in the current efforts for the development of standards for microfluidics.

\section{Acknowledgments}

DRR would like to acknowledge NIST-on-a-Chip funding for the NIST-on-a-Chip Microfluidics Project.

\section{References}

[1] International Organization for Standardization (2016) ISO IWA 23:2016-Interoperability of Microfluidic Devices: Guidelines for Pitch Spacing Dimensions and Initial Device Classification (International Organization for Standardization, Geneva, Switzerland). Available at https://www.iso.org/standard/70603.html

[2] Van Heeren H (2015) Microfluidic survey: Reliability of Microfluidics Based Devices and Components (MFManufacturing website). Available at http://mfmanufacturing.eu/wp-content/uploads/General-results-survey-microfluidic-reliability21/March/2015.pdf

[3] International Organization for Standardization (2009) ISO 10991:2009-Micro Process Engineering — Vocabulary (International Organization for Standardization, Geneva, Switzerland). Available at https://www.iso.org/standard/46546.html

About the authors: Darwin R. Reyes is a biomedical engineer and project leader in the Microsystems and Nanotechnology Division of the Physical Measurement Laboratory at NIST (Gaithersburg, MD). Henne van Heeren is CEO of enablingMNT, based in The Netherlands.

The National Institute of Standards and Technology is an agency of the U.S. Department of Commerce. 